\title{
Effect of Fish Scale Hydrolysed Collagen on Stability of Water-in-Virgin Coconut Oil Emulsion for Potential Topical Application
}

\author{
${ }^{1}$ HasnorIzzati Che Razali, ${ }^{1}$ Ismail Zainol, ${ }^{1}$ Wan Rusmawati Wan Mahamod, ${ }^{2}$ Haniza Hanim Mat Zain, \\ ${ }^{1}$ Norlaili Abu Bakar, ${ }^{1}$ Mohd Anuar Alwi and ${ }^{1}$ Norlizah Jamil \\ ${ }^{1}$ Polymer Laboratory, Department of Chemistry, \\ ${ }^{2}$ Department of Biology, Faculty of Science and Mathematics, University Pendidikan Sultan Idris, \\ 35900 Tanjung Malim, Perak, Malaysia \\ ismail.zainol@fsmt.upsi.edu.my
}

\begin{abstract}
Hydrolysed collagen intake has been shown to have a positive effect on flexibility and elasticity of the skin. However, topical use of hydrolysed collagen in emulsion form is under-explored. The aim of this study was to investigate the effect of hydrolysed collagen existence on the physical stability and droplet size of different formulations of water-in-virgin coconut oil emulsion. The obtained emulsion via. high shear homogenization, both before and after incorporated with hydrolysed collagen showed good physical stability with no separation layersand suitable to be administered on skin. Incorporating hydrolysed collagen caused a small additional decrease in emulsion droplet size compared to typical emulsion. The dispersion of small water-based hydrolysed collagen droplets in emulsion plays an important role for a better permeation of hydrolysed collagen into the skin, once it applied topically. The rheological properties of emulsion attribute to shear thinning behaviour which is appropriate for the needs in the topical application. The results recommend an emulsion system as an innovative approach for enhancing the transdermal penetration of hydrolysed collagen into skin for topical application.
\end{abstract}

Key words: Emulsion, high shear homogenization, hydrolysed collagen, stability, topical application, transdermal penetration

\section{INTRODUCTION}

The demands of collagen as useful biomaterials have been extensively increased in biomedical and cosmetic industries due to its potential in drug delivery, enhanced fibroblast cell proliferation and provided structural support to the skin which can appear youthful (Chak et al., 2013; Kalarikkal et al., 2016 and Borumand and Sibilla, 2014). Collagen is a natural protein in the body that provides a structure to the skin and works hand-in-hand with another protein, called elastinto increase the flexibility of skin as it needs to stretch and return to its original state as the body moves. As one ages, the fibroblasts activities start to slow down in producing new collagen fibres in the body. Therefore, the problem has gained attention among researcher to find alternative source of collagen. It is believed that taking collagen can facilitate the biosynthesis of natural collagen in the body and thus improved the structure of epidermal appearance (Schagen, 2017; Song and Li, 2017). The oral administration of collagen may have a slower effect due to slower release or may quickly pass the metabolism process without been digested thereby entering the rectum as an indigestible molecule waiting to be removed from the body (Alexander et al., 2012; Prausnitz and Langer, 2008). Topical administration allows the collagen targeting directly to the to the dermis layer where the fibroblast cells were placed. However, skin becomes a barrier to protect the body from varied external insults. Therefore, collagen preparation is very important to ensure it will penetrate into the targeted site, thus can provide the desired functionality.

Hydrolysed collagen from fish scales was prepared in this study as an alternative of bovine or porcine source is biocompatible, weak antigenicity and biodegradable (Tang and Saito, 2015; Yamada et al., 2014). The usage of fish scales to derive collagen will not only reduce pollution but may reclaim fish waste to high value added materials. However, low molecular weights $\left(\mathrm{M}_{\mathrm{w}}<1,500 \mathrm{Da}\right)$ of prepared hydrolysed collagen tend to agglomerate

Corresponding Author: Ismail Zainol, Polymer Laboratory, Department of Chemistry, Faculty of Science and Mathematics, Universiti Pendidikan Sultan Idris, 35900 Tanjung Malim, Perak, Malaysis, ismail.zainol@fsmt.upsi.edu.my 
due to strong Van der Waals interactions of fine particles, thus, reduce its penetration capability into skin (Baert et al., 2007; Dumitriu and Popa, 2013). Study on surface morphology of commercialized hydrolysed collagen was observed in the globular groups form proving the occurrence of particles agglomeration (Latip et al., 2015). Therefore, low transdermal penetration efficiency of hydrolysed collagen when administrated topically (Chai et al., 2010). Now a days, emulsion products are typically loaded with a lot of chemical substances to stabilise and preserve them (Juttulapa et al., 2017; Sasseville, 2014).

In this study, water-in-virgin coconut oil emulsion containing hydrolysed collagen was stabilised with small percentage of non-ionic surfactant that adsorb to the surface of droplet, forming a thin coating around the droplet that inhibit their agglomeration by generating repulsive forces between them (Chung and McClements, 2014). It was reported that the non-ionic surfactants was safe to use as ingredient in personal care products and cosmetics because they do not cause negative side effects to the user (Mahdi et al., 2011).

Hydrolysed collagen and Virgin Coconut Oil (VCO) have gained more attention among researchers due to its unique characteristics in delivering lots of benefits to the health and cosmetic industries. It was found that VCO is traditionally used to enhance beauty appearance, moisturizes the skin and treat many skin disorders (Daud, 2008; Mansor et al., 2012). Malaysia remains as one of the top ten coconut producing countries in the world. Therefore, VCO usage is one of the great things that can increase the utilisation of coconut and income of farmers in our country. The stability of hydrolysed collagen was enhanced in water-in-VCO emulsion via. high shear homogenization technique. The physical stability of emulsion was observed and its suitability to be used in topical application was characterized. The collagen prepared along with the VCO should hold high commercial interests due to its double functionality in improvement of facial skin qualities (moisture contents and relative elasticity).

\section{MATERIALS AND METHODS}

Hydrolysed collagen type I was prepared from Tilapia fish scales using combination of hydrothermal extraction with enzymatic hydrolysis (produced in our polymer laboratory) (Izzati et al., 2017). VCO was purchased from Sanggul Biru Enterprise and Azzahra Sdn Bhd (Bangi, Malaysia), respectively. Protease enzyme 2.4L FG was purchased from Brentag specialities (USA) and both non-ionic surfactants of Span 60 (sorbitan monostearate)
Table 1: Compositions of emulsion

\begin{tabular}{lccr}
\hline-1 & Mixed surfactant (Span 60: Tween 60) & Deionized $\mathrm{H}_{2} \mathrm{O}$ & VCO \\
\hline 0.75 & 5 & 74.10 & 20.90 \\
0.83 & 5 & 80.75 & 14.25 \\
0.94 & 5 & 90.25 & 4.75 \\
\hline
\end{tabular}

and Tween 60 (polysorbate 60) were purchased from Sigma-Aldrich, (USA). Deionized water was prepared using easy pure LF, barnstead (USA). All the materials and chemicals were used without further purification.

Preparation of emulsion: The emulsion compositions of mixed span 60: Tween 60/water/VCO systems were selected from a previous study (Alwi, 2013; Jamil, 2012). The emulsion was preparedusing three compositions which are vary in water volume fraction, $\varphi_{\mathrm{w}}$ of $0.75,0.83$ and 0.94 but have afixed Hydrophilic-Lipophilic Balance (HLB) value (5.006) as summarized in Table 1 . The water phase consist of water-based hydrolysed collagen that was prepared by dissolving the hydrolysed collagen in deionized water $(0,5,10,15,20$ and $25 \mathrm{wt} \%)$ prior to mixing with a hydrophobic surfactant, Tween 60 whereas the oil phase consists of a mixture of $\mathrm{VCO}$ and span 60 . Then the water phase was titrated into the VCO phase and homogenized using a digital Ultra-Turrax homogenizer (model T18 from IKA) at 19,000 rpm for $15 \mathrm{~min}$ Schematic illustration of the formation of water-based hydrolysed collagen droplets is shown in Fig. 1. The emulsion samples were kept at $27 \pm 1^{\circ} \mathrm{C}$ for $24 \mathrm{~h}$ and centrifuged for $5 \mathrm{~min}$ at $3000 \mathrm{rpm}$ before any testing was performed. Emulsion that was prepared without hydrolysed collagen acts as a control sample.

Microscopic analysis: An Optical Polarising Microscope, OPM (Eclipse 50i POL, Nikon, USA) was used to observe the microstructure of resulting emulsion. The emulsion was mixed with a few drops of methylene blue, placed on a slide using a spatula and covered with a glass slide. Polarised Light Microscopy (PLM) modes were used to observe microstructure of an emulsion containing hydrolysed collagen $\left(\varphi_{\mathrm{w}}=0.75\right)$.

Droplet size analysis: The droplet size of the freshly prepared emulsion was measured by a Zetasizer Nano ZS (Malvern, UK), based on Photon Correlation Spectroscopy (PCS) techniques. The samples were prepared by diluting approximately $0.005 \mathrm{~g}$ of emulsion in $5 \mathrm{~mL}$ of VCO with gentle stirring. The measurements were done at room temperature.

pH measurement: The $\mathrm{pH}$ of the prepared emulsion was measured by using a $\mathrm{pH}$ meter (Thermo Scientific Orion 2-star benchtop pH meter, Cole-Parmer Instruments, 


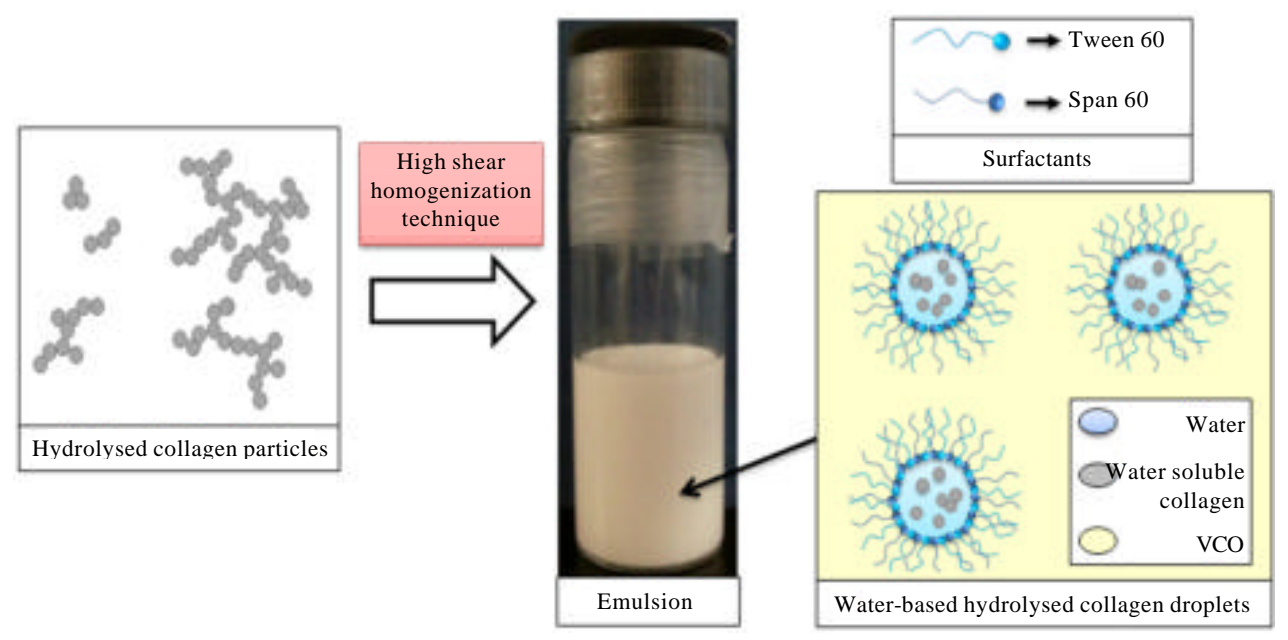

Fig. 1: Schematic illustration of the formation of water-based hydrolysed collagen droplets

USA). The measurement of one sample was repeated three times at $25^{\circ} \mathrm{C}$. The average measurements for each sample were calculated and recorded.

Stability of emulsion: All freshly prepared emulsions were transferred into glass vials and then centrifuged at $3000 \mathrm{rpm}$ for $10 \mathrm{~min}$. After centrifuged, the creaming indexes of all emulsions were measured using the following Eq. 1:

$\begin{aligned} \text { Creaming index }(\%)= & \frac{\text { Height of emulsion layer }(\mathrm{cm})}{\text { Total height of emulsion sample }(\mathrm{cm})} \times \\ & 100 \%\end{aligned}$

Rheological properties: Rheological properties of emulsion were measured by arheometer (Physica MCR 301, Anton Paar, USA) and data were collected in Rheoplus/32 V3.21 Software. A cone and plate system $\left(\mathrm{CP} 25 / 1^{\circ}, 25 \mathrm{~mm}\right)$ was used. A fresh sample was loaded for each run. The thickness of the sample at the centre of the sensor was $0.05 \mathrm{~mm}$. The sample was allowed to rest and adapt for $5 \mathrm{~min}$ before each measurement was performed. All the tests were performed at constant temperature $\left(25^{\circ} \mathrm{C}\right)$. The strain sweep test was measured in the strain ranging from $0.01-100 \%$ at a constant frequency of $1 \mathrm{~Hz}$. Oscillation measurements enable us to obtain the Linear Viscoelastic Region (LVR) and provides the structural properties of each system. The flow characterization of the emulsion samples was obtained by recording shear stress and apparent viscosity values with increasing shearrates ranging from $0.0001-1000 \mathrm{sec}^{-1}$ (logarithmic scale).

\section{RESULTS AND DISCUSSION}

Formation of emulsion: The emulsion does not flow even when the vialis upside down, showing concentrated emulsion produced. Hydrolysed collagen has been successfully loaded up to $20 \mathrm{wt} . \%$ into the water phase of the emulsion system without undergoing any phase change, even after being allowed to stand for $24 \mathrm{~h}$. As was reported by previous study, the greater volume fraction of dispersed phase compared to continued phase with a maximal packing volume has resulted in the formation of a closed-packed droplets structure (Pal, 1999). Concentrated emulsion could retard the free motion of the droplet thereby delaying flocculation and coalescence of the droplets ( $\mathrm{Ng}$ et al., 2014).

Microscopic study: The microscopic images of the typical emulsion and emulsion containing $5 \mathrm{wt} . \%$ of hydrolysed collagen at various $\varphi_{\mathrm{w}}$ of $0.75,0.83$ and 0.94 are shown in Fig. 2. The droplets dispersed widely in emulsion were light blue in color and showed spherical in shape. Since, methylene blue used in preparation of sample analysis is a water-soluble compound, the dispersion can be attributed as a type of water-in-oil (w/o) emulsion. In general, the size of the emulsion droplet containing $5 \mathrm{wt} . \%$ of hydrolysed collagen is much smaller than typical emulsion, irrespective of the water volume fraction studied. The microscopic results also showed that the droplet sizes of emulsions were decreased with increasing $\varphi_{\mathrm{w}}$ of $0.75-0.94$. Similar results have been reported by Anisa and Nour (2010) that the $20-80 \%$ w/o which lower water volume fraction exhibit fine emulsion droplets compared to the $50-50 \% \mathrm{w} / \mathrm{o}$. They also found that the 


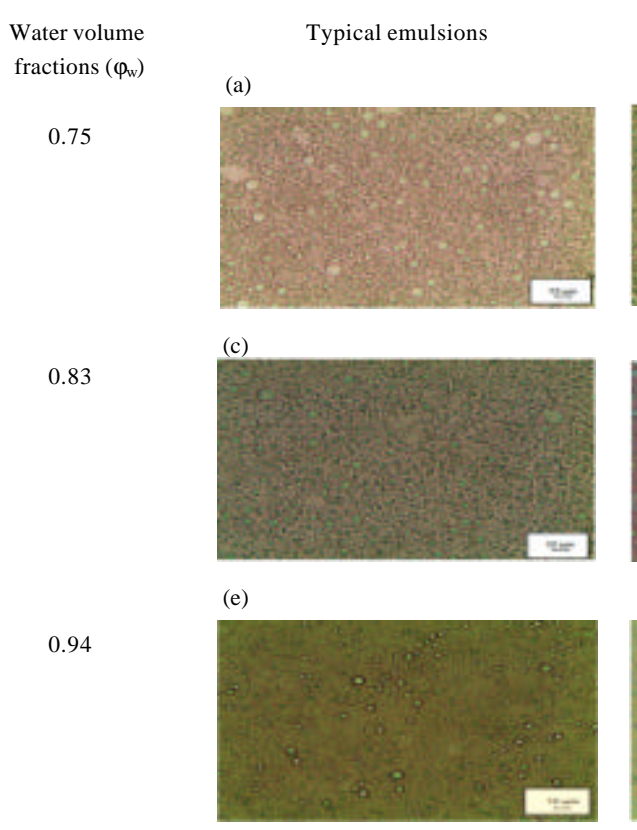

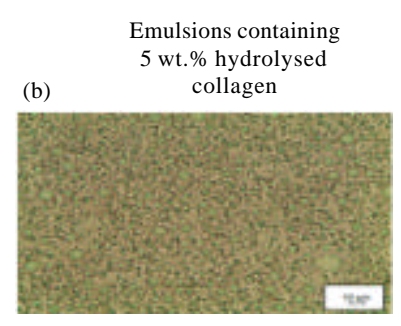

(d)

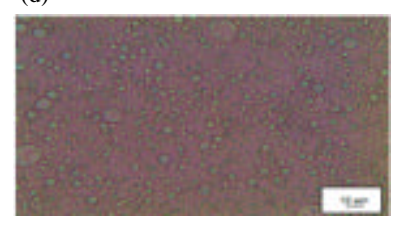

(f)

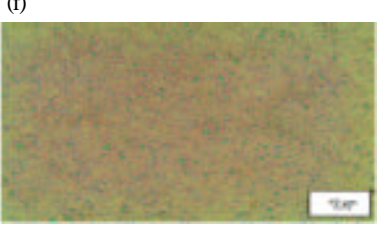

Fig. 2: a-f) Microscopic images of typical emulsion and emulsion containing $5 \mathrm{wt} . \%$ hydrolysed collagen at different water volume fraction, $\varphi_{\mathrm{w}}$ of $0.75,0.83$ and 0.94

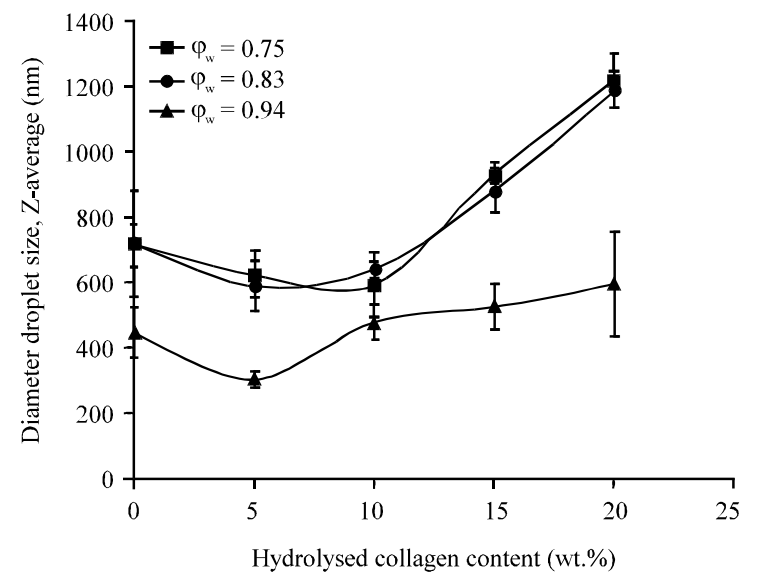

Fig. 3: Droplet size of emulsions at different water volume fractions, $\varphi_{w}$ of $0.75,0.83$ and 0.94 as a function of hydrolysed collagen content

viscosity increased linearly with increasing of $\varphi_{\mathrm{w}}$ have supported the result of the concentrated emulsion prepared in this study. High water volume fraction of the emulsion system leading to decrease in the particle distances as well as an increase of resistance to flow, due to the increase of hydrogen bonds.

Droplet size measurement: The average droplet size (Z-average) of the emulsions at different $\varphi_{w}$ of $0.75,0.83$ and 0.94 as a function of hydrolysed collagen content is shown in Fig. 3. The average droplet size of typical emulsion was found decreased with increasing $\varphi_{w}$. All samples at different $\varphi_{\mathrm{w}}$ exhibit similar trends in terms of droplet size changes against collagen content. The droplet size of the emulsion prepared at higher $\varphi_{\mathrm{w}}$ of 0.83 and 0.94 reduced to a minimum with the addition of hydrolysed collagen as low as $5 \mathrm{wt} \%$. Meanwhile the minimum droplet size of the emulsion prepared at $\varphi_{\mathrm{w}}$ of 0.75 was obtained after adding $10 \mathrm{wt} . \%$ hydrolysed collagen. A strong interaction between adjacent collagen particles caused by hydrogen bonding leads to a decrease in the size of the emulsion droplets, especially, for this closed-packed emulsion structure. The small size of the emulsion droplets allows for easy diffusion through the epidermal barrier when considering application to the skin In this study, the preparation of small and monodisperse droplets of emulsion is the main goal to ensure that the hydrolysed collagen can be absorbed through the skin easily when it was topically applied.

Regardless of the water volume fraction $\left(\varphi_{w}\right)$, all the emulsion droplet sizes increased after further addition of hydrolysed collagen up to $20 \mathrm{wt} . \%$. The results showed that the smallest droplet size of emulsion produced at $\varphi_{w}$ of 0.94 even with the presence of $20 \mathrm{wt} \%$ hydrolysed collagen. The distribution of smaller droplet size can be clearly seen in the microscopic image of emulsion in 
Fig. 2. The minimum emulsion droplet size achieved with the addition of $5 \mathrm{wt} \%\left(\varphi_{\mathrm{w}}=0.83\right.$ and 0.94$)$ and $10 \mathrm{wt} . \%$ $\left(\varphi_{\mathrm{w}}=0.83\right)$ of hydrolysed collagen may be caused by disturbances in the balance of HLB. After a certain amount, the emulsion droplets were not able to accommodate the presence of collagen which is a polar compound that will attract the polar surfactant. This causes a disruption to the interaction between the surfactant molecules at the interface membrane. The phase membrane becomes weaker and the presence of high quantities of collagen particles leads to the enlargement of the emulsion droplet. Previous study was reported that the solute interactions are greater with an increasing hydrophilic chain which leads to an increase in the aqueous solubility of the particles and then adecrease to the driving force of the surfactant to form micelles (Gasic et al., 2002).

pH values: The $\mathrm{pH}$ of a product for skin application is required to be the $\mathrm{pH}$ of skin in order to ensure the product will be well tolerated (Hekimoglu, 2004). It was reported that a $\mathrm{pH}$ range of 5-7 is the level buffered by the skin (Yorgancioglu and Bayramoglu, 2013). The $\mathrm{pH}$ values of the emulsion prepared in this study are in the range of 6.40-7.64. The $\mathrm{pH}$ values of the emulsion containing hydrolysed collagenare relatively low compared to typical emulsion. It is due to the presence of an amino acid chain in the hydrolysed collagen structure. Nevertheless, $\mathrm{pH}$ values around 6.0 are still non-irritating $\mathrm{pH}$ value for the skin (Bernardi et al., 2011).

Phase stability: Even after 12 weeks of storage, the creaming indexes of all samples were maintained at $100 \%$, indicates no phase separation observed during the storage. Both typical emulsion and emulsion with hydrolysed collagen appeared to be stable, irrespective of the water volume fraction $\left(\varphi_{\mathrm{w}}\right)$. According to the results obtained, the formulation used is successful to keep an emulsion stable even with the presence of the hydrolysed collagen. It was indicated that the ratio of surfactant as low as five used in this study is sufficient and able to produce a stable emulsion containing hydrolysed collagen up to $20 \mathrm{wt} \%$. It was reported that the emulsifying properties of collagen also play an important role in stability of oil-in-water emulsion by reducing oil droplet size (De Castro Santana et al., 2012).

\section{Rheological properties}

Strain sweep test: Strain sweep tests have been performed on both samples, typical emulsion and emulsion containing 5 wt.\% of hydrolysed collagenas shown in Fig. 4a, b, respectively. All emulsion sowned a higher value of "G than G" within the Linear Viscoelastic Region (LVR) which is the typical behaviour of viscoelastic samples. Although, the range of LVR for both emulsions is almost the same but the downward trend of $G^{\prime}$ for the emulsion containing hydrolysed collagen is quite slow compared to the typical emulsion. This indicates that the emulsion containing collagen is more easily distupted. In addition, the decreasing trend of G' for that emulsion is observed after the LVR value compared to the typical emulsion. This shows that the disruption that occurs in the structure of the water-based hydrolysed collagen droplets caused the decrease in viscosity at high strain amplitude.

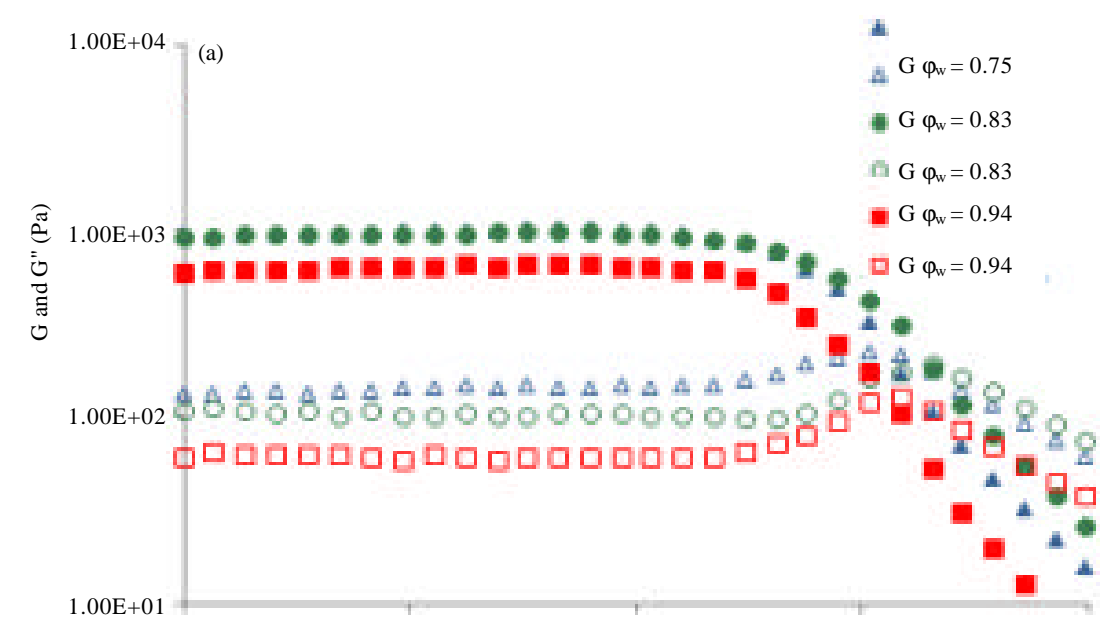

Fig. 4: Continue 


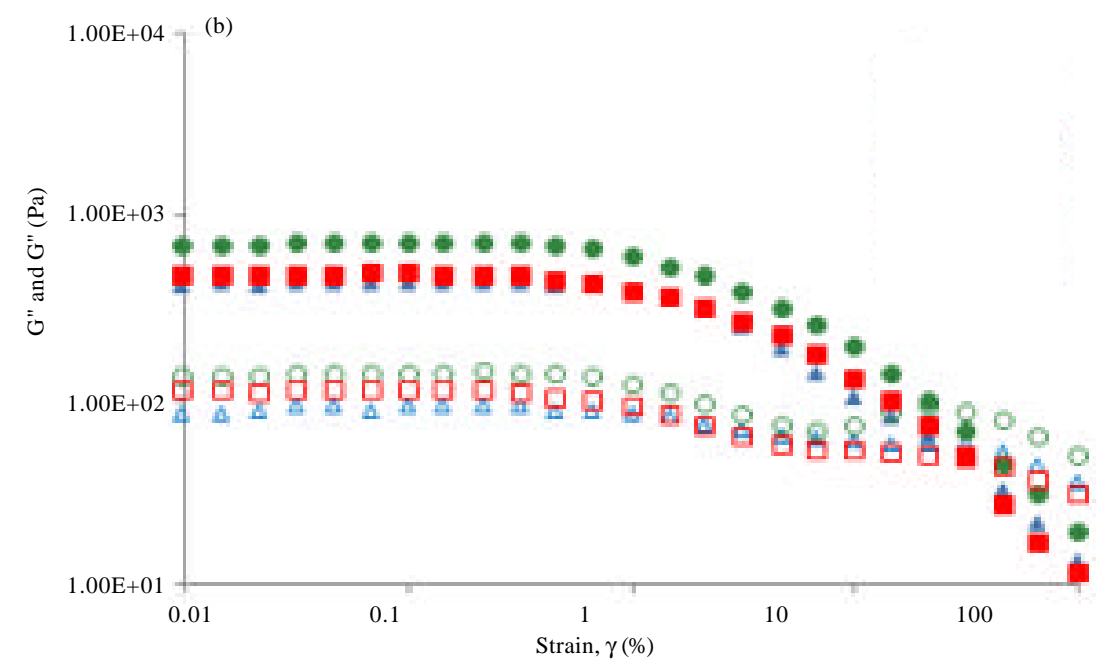

Fig. 4: "G \& G" comparison of; a) Typical emulsion and b) Emulsion containing 5 wt. $\%$ hydrolysed collagen

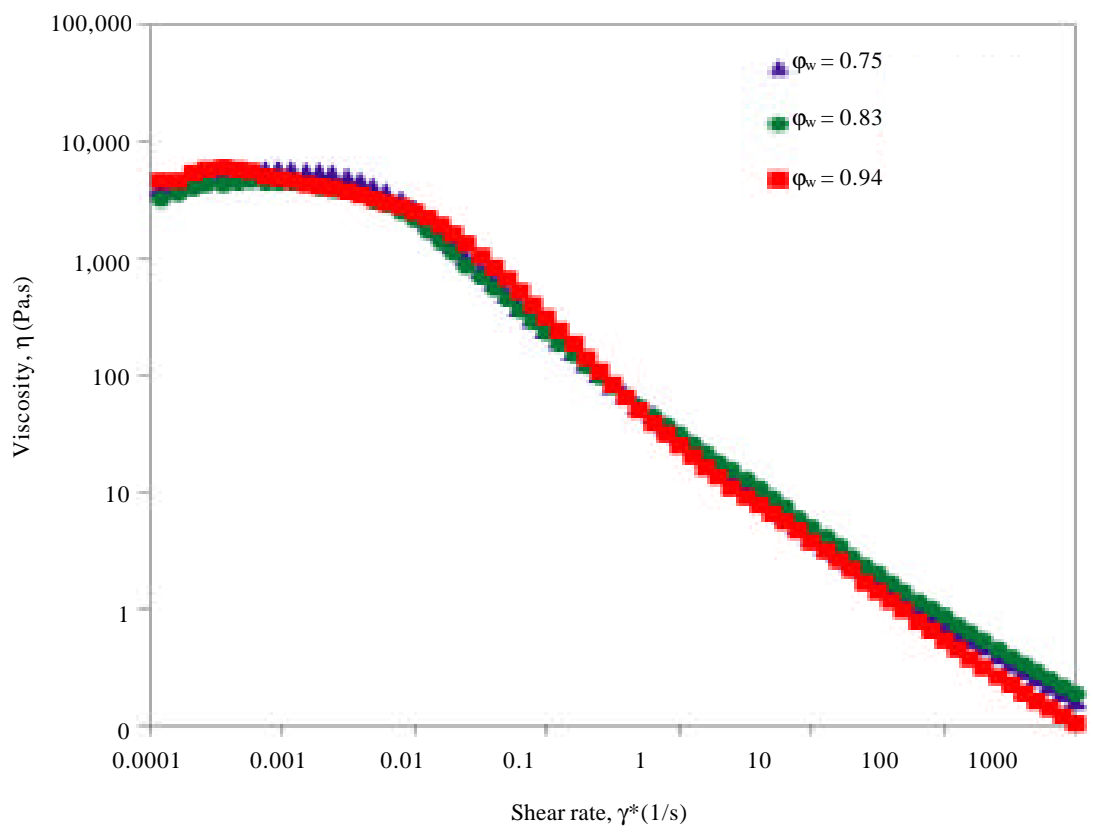

Fig. 5: Flow curve of the emulsion containing 5 wt. $\%$ of hydrolysed collagen at $\varphi_{\mathrm{w}}$ of $0.75,0.83$ and 0.94

However, the strength of the internal structure in an emulsion can be measured by $\tan \delta=$ "G/G" due to its dependent on the differences between "G and G". A clear comparison can be seen from the summary of the G' and Tan $\delta$ values of all emulsions in Table 2. Although, the presence of hydrolysed collagen increases the Tan $\delta$ value of emulsion, reconfirming the occurrence of a small disruption in the internal structure but there was no significant difference in the $\tan \delta$ value of all emulsions studied against the content of hydrolysed collagen. The value of Tan $\delta$ for all samples prepared can still be considered small as it is $>0.3$ Table 2. Liu et al. (2007) was stated that the Tan $\delta$ value of emulsion lower than one exhibited solid-like behavior. This implies that the emulsion is constructed of strong internal interactions (Fig. 5).

Shear flow test: The continuous shear flow test provides important information regarding the ways in which the structure changes to comply with the applied shear 
Table 2: Storage modulus $(\mathrm{G})$ and $\tan \delta$ values of emulsion at $0.1 \%$ strain

\begin{tabular}{lccr}
\hline$\delta_{w}$ & $\begin{array}{c}\text { Hydrolysed collagen in } \\
\text { water phase (wt. \%) }\end{array}$ & $\begin{array}{c}\text { Storage modulus, } \\
\mathrm{G}(\mathrm{Pa})\end{array}$ & $\operatorname{Tan} \delta$ \\
\hline 0.75 & 0 & 969 & 0.15 \\
& 5 & 434 & 0.20 \\
& 10 & 1080 & 0.25 \\
& 15 & 1970 & 0.27 \\
0.83 & 20 & 2540 & 0.23 \\
& 0 & 962 & 0.11 \\
& 5 & 724 & 0.20 \\
& 10 & 1940 & 0.25 \\
& 15 & 3870 & 0.29 \\
0.94 & 20 & 1310 & 0.27 \\
& 0 & 631 & 0.09 \\
& 5 & 479 & 0.23 \\
& 10 & 867 & 0.28 \\
& 15 & 664 & 0.27 \\
& 20 & 1640 & 0.27 \\
\hline
\end{tabular}

flow in different conditions such as storage, processing and application. The flow curve in Fig. 5 shows emulsion containing 5 wt.\% hydrolysed collagen exhibited non-Newtonian characteristics, since, the emulsion underwent deformation when subjected to the shearing tension test and did not exhibit constant viscosity. The emulsion behaved as a pseudoplastic or with shear-thinning as the emulsion viscosity decreases with shear. The shear rate remains at zero until a threshold shear stress is reached and the pseudoplastic sample flow begins. Such, behaviour is due to the existence of an intermolecular network which initially acts like a solid and becomes a fluid when the applied stress exceeds the strength of the network. Shear thinning behaviour is the most relevant criteria for an application in the cosmetic field (Kwak et al., 2015).

The rheological behaviour of the emulsion in shear flow test also depends on the concentration of the dispersed phase (Foudazi et al., 2012). It was clearly shown that the flow properties of the emulsion changed drastically with increasing shear. This has proved that the emulsion droplets are densely packed and easy to deform. The emulsion prepared at the highest water volume fraction $\left(\varphi_{\mathrm{w}}=0.94\right)$ has the highest viscosity at low shear. This could be confirmed by the droplet size measurement, in which the emulsion droplets prepared at $\varphi_{\mathrm{w}}$ of 0.94 shows the smallest size than the others. Similar results have been reported by Malkin et al. (2004) that the emulsion with small droplet size exhibited high viscosity at a low shear rate.

\section{CONCLUSION}

A stable water-in-oil emulsion containing fish scale hydrolysed collagen was successfully prepared via. high shear homogenization technique. The hydrolysed collagen can be uploaded up to $20 \mathrm{wt} \%$ into an emulsion system of span $60 / \mathrm{VCO}-\mathrm{Tween} 60 / \mathrm{H}_{2} \mathrm{O}$ at $\varphi_{\mathrm{w}}$ of $0.75,0.83$ and 0.94 without phase separation. A high shear homogenization weakens the intermolecular interaction of hydrolysed collagen particles and forms the high dispersion of water-based hydrolysed collagen droplets in virgin coconut oil phase. Aminimum droplet size (Z-average) of $305 \mathrm{~nm}$ was obtained after loaded with 5 wt. $\%$ hydrolysed collagen at $\varphi_{\mathrm{w}}$ of 0.94 . All results obtained show that the emulsion system is an ideal medium for the dispersion of hydrolysed collagen particles. The small size of water-based hydrolysed collagen droplets will allow the easily diffusion of hydrolysed collagen particles through the skin. This finding also showed that the $\mathrm{pH}$ of the emulsionis acceptable for topical application. In addition, the emulsion prepared has pseudoplastic and shear-thinning characteristics that are suitable for cosmetic application such as creams and moisturizers. Emulsion containing hydrolysed collagen has a potential to bedirectly applied to the skin. The addition of hydrolysed collagen as an active compound in the emulsion system can give value added to the VCO-based emulsion. As this is a preliminary study, further studies will include in vitro and in vivo evaluation for topical delivery.

\section{ACKNOWLEDGEMENT}

Financial assistance from the FRGS grant (2013-0187102-020), GPU grant (2013-0069-102-01), GPU grant (2016-0183-102-01), MyBrain scholarship and facilities support from the Universiti Pendidikan Sultan Idris are gratefully acknowledged.

\section{REFERENCES}

Alexander, A., S. Dwivedi, Ajazuddin, T.K. Giri, S. Saraf, S. Saraf and D.K. Tripathi, 2012. Approaches for breaking the barriers of drug permeation through transdermal drug delivery. J. Controlled Release, 164: 26-40.

Alwi, A., 2013. Formulation of cosmetic emulsion based VCO from mixed span 60 and tween series ( $t 60$, t80, t81, t85). BSc Thesis, Universiti Pendidikan Sultan Idris, Tanjong Malim, Malaysia.

Anisa, A.N.I. and A.H. Nour, 2010. Affect of viscosity and droplet diameter on water-in-oil (w/o) emulsions: An experimental study. World Acad. Sci. Eng. Technol., 4: 595-598. 
Baert, B., E. Deconinck, M. Van Gele, M. Slodicka and P. Stoppie et al., 2007. Transdermal penetration behaviour of drugs: CART-clustering, QSPR and selection of model compounds. Bioorg. Med. Chem., 15: 6943-6955.

Bernardi, D.S., T.A. Pereira, N.R. Maciel, J. Bortoloto and G.S. Viera et al., 2011. Formation and stability of oil-in-water nanoemulsions containing rice bran oil: in vitro and in vivo assessments. J. Nanobiotechnol., 9: 1-9.

Borumand, M. and S. Sibilla, 2014. Daily consumption of the collagen supplement Pure Gold Collagen ( reduces visible signs of aging. Clin. Interventions Aging, 9: 1747-1758.

Chai, H.J., J.H. Li, H.N. Huang, T.L. Li and Y.L. Chan et al., 2010. Effects of sizes and conformations of fish-scale collagen peptides on facial skin qualities and transdermal penetration efficiency. J. Biomed. Res., 2010: 1-9.

Chak, V., D. Kumar and S. Visht, 2013. A review on collagen based drug delivery systems. Intl. J. Pharm. Teach. Pract., 4: 811-820.

Chung, C. and D.J. McClements, 2014. Structure-function relationships in food emulsions: Improving food quality and sensory perception. Food Struct., 1: 106-126.

Daud, Z., 2008. [What and Why-Virgin Coconut Oil]. 2nd Edn., Klapa Biotech Corporation, Selangor, Malaysia, (In Malay).

De Castro Santana, R., A.C.K. Sato and R.L. da Cunha, 2012. Emulsions stabilized by heat-treated collagen fibers. Food Hydrocolloids, 26: 73-81.

Dumitriu, S. and V. Popa, 2013. Polymeric Biomaterials: Structure and Function. Vol. 1, CRC Press Publishing Company, Boca Raton, Florida, USA., ISBN:9781420094718, Pages: 920.

Foudazi, R., I. Masalova and A.Y. Malkin, 2012. The rheology of binary mixtures of highly concentrated emulsions: Effect of droplet size ratio. J. Rheol., 56: 1299-1301.

Gasic, S., B. Jovanovi and S. Jovanovi, 2002. The stability of emulsions in the presence of additives. J. Serb. Chem. Soc., 67: 31-39.

Hekimoglu, S., 2004. Effectiveness of cosmetic. Cosmet. Sci., 1: 313-331.

Izzati, H., I. Zainol and H. Hanim, 2017. Low molecular weight collagen from Tilapia fish scales for potential cosmetic application. Der Pharma. Chem., 9: 108-144.

Jamil, N., 2012. Characterization and stability of concentrated cosmetics emulsion of w/o based VCO. BSc Thesis, Sultan Idris Education University, Perak Darul Ridzuan, Malaysia.
Juttulapa, M., S. Piriyaprasarth, H. Takeuchi and P. Sriamornsak, 2017. Effect of high-pressure homogenization on stability of emulsions containing zein and pectin. Asian J. Pharm. Sci., 12: 21-27.

Kalarikkal, N., R. Augustine, O.S. Oluwafemi, K.S. Joshy and S. Thomas, 2016. Nanomedicine and Tissue Engineering: State of the Art and Recent Trends. Apple Academic Press Inc., Florida, USA., ISBN:9781498726429, Pages: 562.

Kwak, M.S., H.J. Ahn and K.W. Song, 2015. Rheological investigation of body cream and body lotion in actual application conditions. Korea Australia Rheol. J., 27: 241-251.

Latip, L.D., W. Zzaman, M.Z. Abedin and T.A. Yang, 2015. Optimization of spray drying process in commercial hydrolyzed fish scale collagen and characterization by scanning electron microscope and fourier transform infrared spectroscopy. J. Food Process. Preserv., 39: 1754-1761.

Liu, H., Xu, X. M. and S.D. Guo, 2007. Rheological, texture and sensory properties of low-fat mayonnaise with different fat mimetics LWT-Food Sci. Technol., 40: 946-954.

Mahdi, E.S., M.H.F. Sakeena, M.F. Abdulkarim, G.Z. Abdullah and M.A. Sattar et al., 2011. Effect of surfactant and surfactant blends on pseudoternary phase diagram behavior of newly synthesized palm kernel oil esters. Drug Des. Dev. Ther., 5: 311-323.

Malkin, A. Y., I. Masalova, P. Slatter and K. Wilson, 2004. Effect of droplet size on the rheological properties of highly-concentrated w/o emulsions. Rheol. Acta, 43: 584-591.

Mansor, T.S.T., Y.B. Che Man, M. Shuhaimi, M.J. Abdul Afiq and F.K.M. Ku Nurul, 2012. Physicochemical properties of virgin coconut oil extracted from different processing methods. Intl. Food Res. J., 19: 837-845.

Ng, S.P., O.M. Lai, F. Abas, H.K. Lim and C.P. Tan, 2014. Stability of a concentrated oil-in-water emulsion model prepared using palm olein-based diacylglycerol/virgin coconut oil blends: Effects of the rheological properties, droplet size distribution and microstructure. Food Res. Intl., 64: 919-930.

Pal, R., 1999. Yield stress and viscoelastic properties of high internal phase ratio emulsions. Colloid Polym. Sci., 277: 583-588.

Prausnitz, M.R. and R. Langer, 2008. Transdermal drug delivery. Nat. Biotechnol., 26: 1261-1268.

Sasseville, D., 2014. Hypersensitivity to preservatives. Dermatologic Ther., 17: 251-263. 
Schagen, S.K., 2017. Topical peptide treatments with effective anti-aging results. Cosmet., 4: 1-14.

Song, H. and B. Li, 2017. Beneficial effects of collagen hydrolysate: A review on recent developments. Biomed J. Sci. Technol. Res., 1: 1-4.

Tang, J. and T. Saito, 2015. Biocompatibility of novel type I collagen purified from tilapia fish scale: An in vitro comparative study. BioMed. Res. Intl., 2015: $1-8$.
Yamada, S., K. Yamamoto, T. Ikeda, K. Yanagiguchi and Y. Hayashi, 2014. Potency of fish collagen as a scaffold for regenerative medicine. BioMed. Res. Intl., 2014: 1-8.

Yorgancioglu, A. and E.E. Bayramoglu, 2013. Production of cosmetic purpose collagen containing antimicrobial emulsion with certain essential oils. Ind. Crops Prod., 44: 378-382. 